QUARTERLY OF APPLIED MATHEMATICS

VOLUME LXIV, NUMBER 2

JUNE 2006, PAGES 229-252

$\mathrm{S} 0033-569 \mathrm{X}(06) 01001-2$

Article electronically published on April 17, 2006

\title{
A WAY TO COMPUTE THE GRAVITATIONAL POTENTIAL FOR NEAR-SPHERICAL GEOMETRIES
}

\author{
BY \\ PAVEL GRINFELD (Department of Mathematics, Drexel University, Philadelphia, Pennsylvania) \\ AND \\ JACK WISDOM (Department of EAPS, MIT, Cambridge, Massachusetts)
}

\begin{abstract}
We use a boundary perturbation technique based on the calculus of moving surfaces to compute the gravitational potential for near-spherical geometries with piecewise constant densities. The perturbation analysis is carried out to third order in the small parameter. The presented technique can be adapted to a broad range of potential problems including geometries with variable densities and surface density distributions that arise in electrostatics. The technique is applicable to arbitrary small perturbations of a spherically symmetric configuration and, in principle, to arbitrary initial domains. However, the Laplace equation for an arbitrary domain can usually be solved only numerically. We therefore concentrate on spherical domains which yield a number of geophysical applications.

As an illustration, we apply our analysis to the case of a near spherical triaxial ellipsoid and show that third order estimates for ellipticities such as that of the Earth are accurate to ten digits. We include an appendix that contains a concise, but complete, exposition of the tensor calculus of moving interfaces.
\end{abstract}

1. Introduction. This paper demonstrates how to use the calculus of moving surfaces to compute the gravitational potential for near spherical geometries based on a perturbation theory approach. Our expressions apply equally well (with a minus sign!) to the electrostatic potential when no surface charges are present.

The perturbation of the potential is induced by a small deformation of the boundary of the domain. Our analysis applies to an arbitrary sufficiently smooth small deformation. We consider the case of constant density which can be extended to piecewise constant by the superposition principle. We do not consider the case of varying density, since the most challenging and interesting part of the analysis deals with the density discontinuities at the boundary of the domain. The presented technique can also be applied to boundary density distributions that arise in the analysis of the electrostatic potential of a conductor.

Received March 1, 2005.

2000 Mathematics Subject Classification. Primary 81V17.

(C)2006 Brown University 


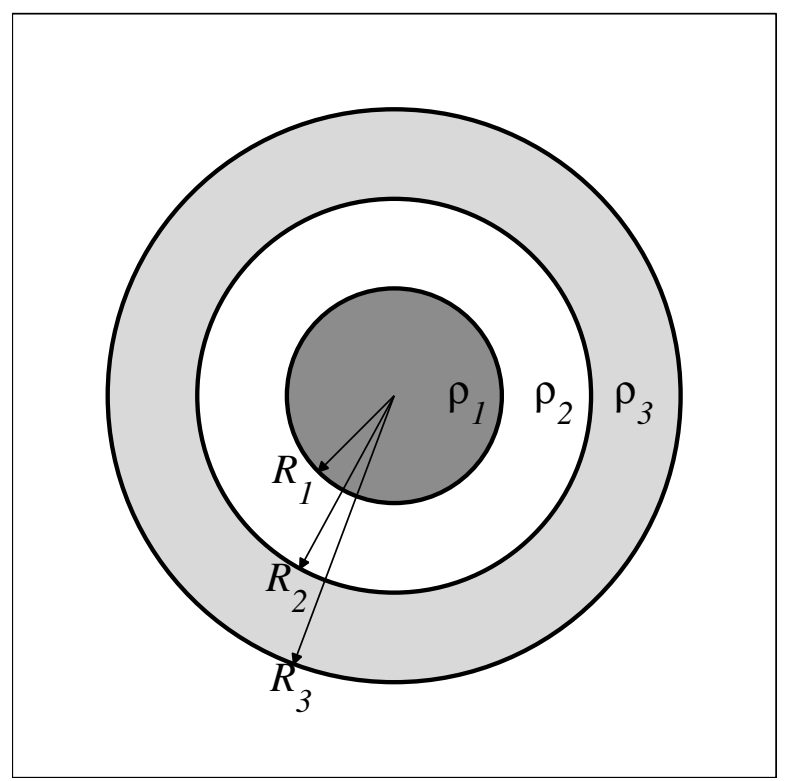

FIG. 1. A spherically symmetric "unperturbed" configuration.

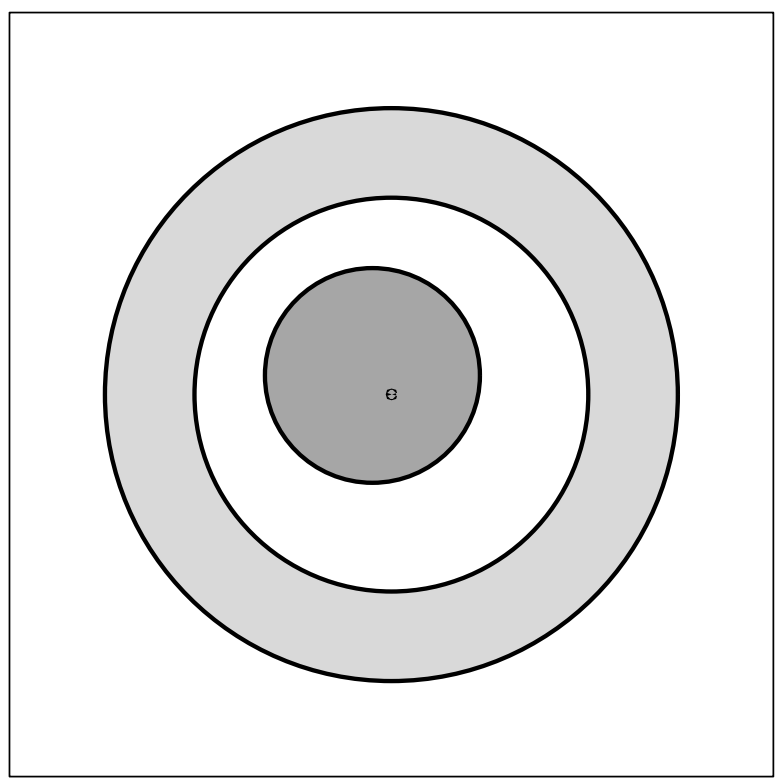

FIG. 2. The spherically symmetric configuration is perturbed by a slight shift of the inner sphere. Such perturbations arise in the analysis of the Slichter modes of a planet. 


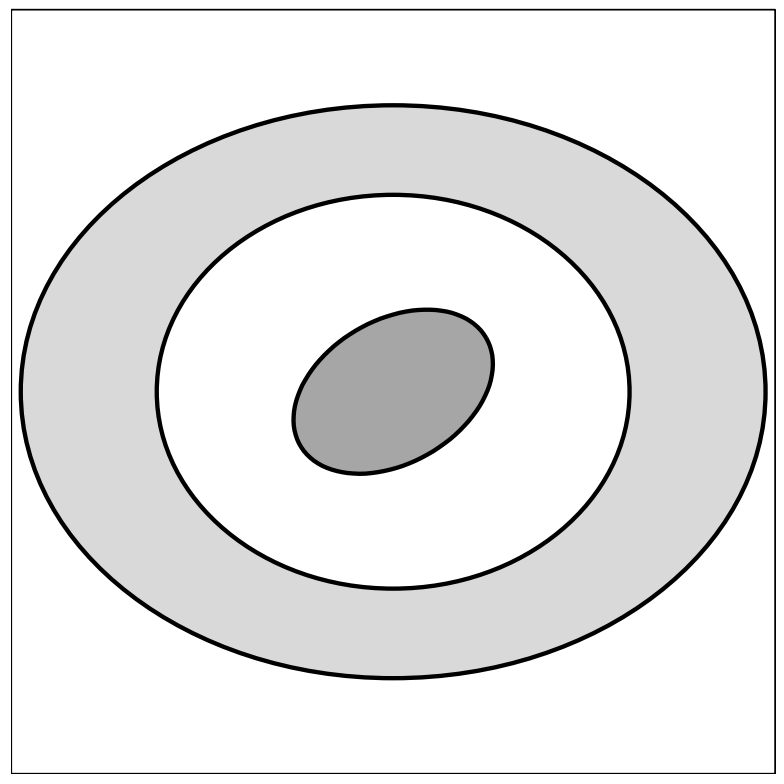

FIG. 3. An ellipsoidal perturbation of the spherically symmetric configuration. Such perturbations find applications in the analysis of the librational modes of an ellipsoidal planet. Note that the inner sphere can be arbitrarily turned relative to the shell.

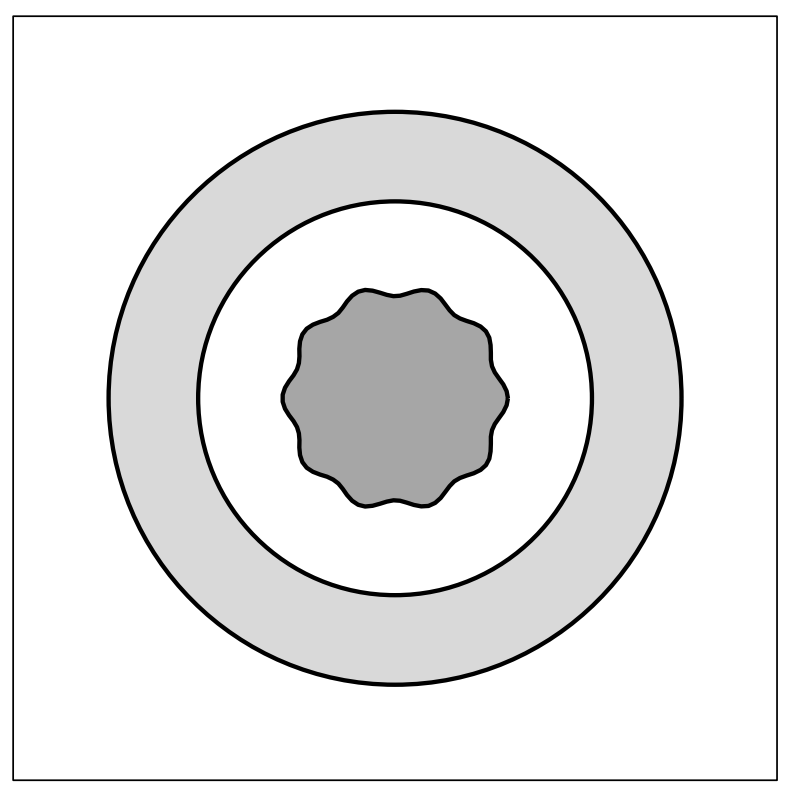

FIG. 4. An arbitrary small perturbation of the spherically symmetric configuration. Such perturbations may arise in the study of phase transformations at the inner core boundary of a planet. 
The presented analysis has a number of geophysical applications:

a. The study of Slichter modes of the inner core. First studied in 1961 [9] by Slichter, the modes refer to the translational motion of the inner core of a planet with respect to the outer core and the mantle. Busse [1] presented an analytical treatment of this problem in which he treated the constituents of the planet as constant density (Figure 11). A small translational displacement of the inner core (Figure 22) can be treated as a small boundary perturbation of the unperturbed spherical configuration.

b. Libration of the inner core. An inner core and a mantle which are slightly ellipsoidal (Figure 3) are coupled by a gravitational torque that is roughly proportional to the ellipticities. As a result, the two parts of the planet oscillate about a stable equilibrium configuration. Because the product of the (small) ellipticities is involved, the effect is second order and requires a second order perturbation analysis.

c. Phase transformations at the inner core boundary. It is a well-accepted hypothesis that the matter at the inner core - outer core boundary undergoes phase transformations [4. If the inner core is no longer assumed rigid, then potentially arbitrary deformations of the interface may occur, as illustrated in the exaggerated Figure 4 , and a technique flexible enough to analyze such deformations is required.

\section{Methodology.}

2.1. A word about our notation. Throughout the paper we adhere to the indicial notation of tensor calculus, [7, [6], [12. In our experience, this language is best for the calculus of moving surfaces outlined in the Appendix. For the sake of consistency we use indicial expressions such as $N^{i} \nabla_{i} \psi$ even where the more conventional $\mathbf{N} \cdot \nabla \psi$ would have been sufficient.

2.2. Evolution of geometries and the power series. The gravitational potential field is determined by the distribution of mass densities. Since we consider piecewise constant densities, the potential field is essentially determined by the geometry of the configuration. We suppose that the geometry $\Omega^{\prime}$, for which the potential field is to be computed, is close to a spherically symmetric configuration $\Omega$. Then the desired gravitational potential $\psi^{\prime}(z)$ for $\Omega^{\prime}$ is close to $\psi_{0}(z)$ for $\Omega$. The letter $z$ indicates a point in the three-dimensional space.

We imagine a smooth evolution $\Omega(\tau)$ of $\Omega$ into $\Omega^{\prime}$ indexed by a time-like parameter $\tau$ (sometimes conveniently thought of as having dimensions of time) such that

$$
\begin{aligned}
& \Omega(0)=\Omega, \\
& \Omega(1)=\Omega^{\prime} .
\end{aligned}
$$

The gravitational potential $\psi$ also undergoes a smooth evolution $\psi(\tau, z)$ such that

$$
\begin{aligned}
& \psi(0, z)=\psi_{0}(z), \\
& \psi(1, z)=\psi^{\prime}(z) .
\end{aligned}
$$

We approximate $\psi^{\prime}(z)$ by the first $n$ terms of the power series

$$
\psi^{\prime}(z) \approx \psi_{0}(z)+\left.\frac{\partial \psi(\tau, z)}{\partial \tau}\right|_{\tau=0}+\left.\frac{1}{2} \frac{\partial^{2} \psi(\tau, z)}{\partial \tau^{2}}\right|_{\tau=0}+\ldots+\left.\frac{1}{N !} \frac{\partial^{N} \psi(\tau, z)}{\partial \tau^{N}}\right|_{\tau=0}
$$


and calculate the first three terms explicitly. Note that by letting $\Delta \tau=1$, we consider slow evolutions over a finite period of time rather than an equivalent scenario with finite velocities over a short period of time.

2.3. Two approaches to the problem. Our task is to compute $\partial^{n} \psi / \partial \tau^{n}$ at $\tau=0$. At a given time $\tau$, the potential is given by the conceptual integral

$$
\psi(\tau, z)=-\int_{\Omega} \frac{\rho\left(\tau, z^{*}\right)}{\operatorname{Distance}\left(z, z^{*}\right)} d \Omega^{*},
$$

where $\rho(\tau, z)$ is the imaginary evolution of density distribution. This integral can in principle be differentiated with respect to $\tau$ to obtain $\partial \psi^{n} / \partial \tau^{n}$. Since this approach requires a complicated analysis of integrals with singularities, we prefer a different approach based on Poisson's equation.

Away from the surfaces of density discontinuities, the gravitational potential $\psi$ satisfies Poisson's equation

$$
\nabla^{i} \nabla_{i} \psi=4 \pi G \rho .
$$

In addition, $\psi$ vanishes at infinity and is finite everywhere. Finally, across the interfaces $S$ of density discontinuities, $\psi$ is continuous along with its derivatives

$$
\begin{aligned}
{[\psi]_{S} } & =0, \\
N^{i}\left[\nabla_{i} \psi\right]_{S} & =0,
\end{aligned}
$$

where

$$
[X]_{S}
$$

stands for the jump in quantity $X$ across the surface $S$. We require continuity only for the normal derivatives since the continuity of tangential derivatives follows from the continuity of $\psi$.

2.4. Outline of the procedure. We start by calculating the unperturbed potential $\psi_{0}(r)$ for the initial domain $\Omega$. Since $\Omega$ is spherically symmetric, a sphere of radius $R$ in our case, this is a straightforward task. We then derive a system of equations for $\partial^{n} \psi / \partial \tau^{n}$ by repeatedly differentiating (or perturbing) the system (77)-(9) with respect to $\tau$. Differentiating the bulk condition (7) $n$ times yields

$$
\nabla^{i} \nabla_{i} \frac{\partial^{n} \psi}{\partial \tau^{n}}=0
$$

The derivative of the right-hand side of (7) vanishes since $\rho$ is presumed piecewise constant.

The differentiation of the boundary conditions (8) and (9) is far more challenging and requires an application of the so-called $\delta / \delta \tau$-derivative, a key operation in the calculus of moving surfaces (see the Appendix).

The perturbed system needs to be solved for the initial configuration since the power series (5) requires the values of $\partial^{n} \psi / \partial \tau^{n}$ at $\tau=0$. The spherical symmetry of the initial configuration allows us to solve the resulting systems by separation of variables.

We consider a single domain of density $\rho$. More complicated geometries with piecewise constant densities can be obtained by superimposing solid domains. For example, a planet with an inner core of density $\rho_{1}$, an outer core of density $\rho_{2}$ and a mantle of 
density $\rho_{3}$ can be represented as a sphere of radius $\rho_{3}$ with a smaller sphere (outer core) of density $\rho_{2}-\rho_{3}$ and an even smaller sphere (inner core) of density $\rho_{1}-\rho_{2}$.

For a sphere of radius $R$ and density $\rho$, the unperturbed potential $\psi_{0}(z)$ is given by

$$
\psi_{0}(r)=4 \pi G \rho \frac{R^{2}}{3}\left\{\begin{array}{cc}
\frac{1}{2}\left(\frac{r}{R}\right)^{2}-\frac{3}{2}, & z<\Omega, \\
-\left(\frac{r}{R}\right)^{-1}, & z>\Omega,
\end{array}\right.
$$

where $z<\Omega$ and $z>\Omega$ is a shorthand notation for "points inside $\Omega$ " and "points outside $\Omega$.

\section{First perturbation of the potential.}

3.1. Solution of Laplace's equation for a sphere. We noted that $\partial^{n} \psi / \partial \tau^{n}$ satisfies Laplace's equation in $\mathbb{R}^{3}$, i.e. $\partial^{n} \psi / \partial \tau^{n}$ is harmonic. Here, we present the general solution for the Laplace equation in $\mathbb{R}^{3}$ that is finite at the origin, vanishes at infinity, and satisfies the given boundary condition on the sphere of radius $R$.

Suppose that $\chi$ is a harmonic function

$$
\nabla^{i} \nabla_{i} \chi=0
$$

and that, in addition to being finite at the origin and zero at infinity, $\chi$ satisfies the following discontinuity conditions across $S$, the boundary of $\Omega$ :

$$
\begin{aligned}
{[\chi]_{S} } & =J, \\
N^{i}\left[\nabla_{i} \chi\right]_{S} & =\frac{H}{R} .
\end{aligned}
$$

Both $J$ and $H$ are determined by the evolution $\Omega(\tau)$, and the factor of $R^{-1}$ was introduced for nondimensionalization. If the fields $J$ and $H$ are represented as a series in spherical harmonics $Y_{l m}(\theta, \phi)$

$$
\begin{aligned}
J & =J^{l m} Y_{l m}(\theta, \phi), \\
H & =H^{l m} Y_{l m}(\theta, \phi),
\end{aligned}
$$

then $\chi$ is given by

$$
\chi(r, \theta, \phi)=\left\{\begin{array}{ll}
\frac{(l+1) J^{l m}+H^{l m}}{2 l+1}\left(\frac{r}{R}\right)^{l}, & z<\Omega \\
\frac{-l J^{l m}+H^{l m}}{2 l+1}\left(\frac{r}{R}\right)^{-l-1}, & z>\Omega
\end{array} \mid Y_{l m}(\theta, \phi) .\right.
$$

We usually prefer complex spherical harmonics $Y_{l m}(\theta, \phi)$ that are normalized to unity on the unit sphere

$$
\int_{|r|=1} Y_{l_{1} m_{1}}(\theta, \phi) Y_{l_{2} m_{2}}^{*}(\theta, \phi) d S=\delta_{l_{1} l_{2}} \delta_{m_{1} m_{2}},
$$

where $*$ means complex conjugation.

As we compute successive perturbations of the system (7)-(9) we obtain Laplace systems of the type (13)-14b), each with its own $H$ and $J$. Denote the $H$ and $J$ appearing 
in the $n$-th perturbation by $J_{n}$ and $H_{n}$ and their spherical harmonic expansion coefficients by $H_{n}^{l m}$ and $J_{n}^{l m}$. The power series approximation of the perturbation $\Delta \psi(r, \theta, \phi)$ of $\psi(r, \theta, \phi)$ (5) is then given by

$$
\Delta \psi(r, \theta, \phi) \approx \sum_{n=1}^{N}\left\{\begin{array}{ll}
\frac{(l+1) J_{n}^{l m}+H_{n}^{l m}}{2 l+1) n !}\left(\frac{r}{R}\right)^{l}, & z<\Omega \\
\frac{-l J_{n}^{l m}+H_{n}^{l m}}{(2 l+1) n !}\left(\frac{r}{R}\right)^{-l-1}, & z>\Omega
\end{array} \mid Y_{l m}(\theta, \phi) .\right.
$$

The task that remains is to determine the surface fields $J_{n}$ and $H_{n}$ and to represent them with respect to the spherical harmonics.

3.2. The $\frac{\delta}{\delta \tau}$-derivative and the interface velocity $C$. This paper relies heavily on the calculus of moving surfaces, whose concise but complete exposition can be found in the Appendix. This section briefly introduces the main concepts, enabling the reader to see the big picture presented in the following section while using the Appendix as a reference.

The two primary concepts on the calculus of moving surfaces is the $\delta / \delta \tau$-derivative and the interface velocity $C$. In the following sections, the boundary conditions for the potential field perturbations are stated only in terms of this operator and this quantity, plus the stationary surface fields such as the mean curvature. Therefore, while the derivation of the perturbation equations requires the knowledge of the calculus of moving surfaces, the interpretation of the resulting equations only requires the concepts introduced in this section.

Consider a one parameter family of curves $S_{\tau}$ indexed by a time-like parameter $\tau$. The family $S_{\tau}$ can also be thought of as a time evolution of a single curve $S$. Let $T\left(\tau, S_{\tau}\right)$ be a scalar field defined on $S_{\tau}$, so $T$ not only changes its values with time but also experiences a change in its domain of definition.

We present a geometric definition of the $\delta / \delta \tau$-derivative at a point $\xi$ on the surface $S_{\tau}$ at time $\tau$, illustrated in Figure 5. Consider two locations of the surface $S_{\tau}$ and $S_{\tau^{*}}$ at nearby times $\tau$ and $\tau^{*}$. Draw the straight line orthogonal to $S_{\tau}$ passing through the point $\xi$. Mark the point $\xi^{*}$, where this straight line intersects $S_{\tau^{*}}$. Define

$$
\frac{\delta T(\tau, \xi)}{\delta \tau}=\lim _{\tau^{*} \rightarrow \tau} \frac{T\left(\tau^{*}, \xi^{*}\right)-T(\tau, \xi)}{\tau^{*}-\tau} .
$$

An equivalent algebraic definition of the $\delta / \delta \tau$-derivative of a scalar is given by equation (108) of the Appendix. A general definition applicable to tensors of arbitrary shape is given by equation (111).

Let $\Delta \mathbf{z}$ be the vector connecting the point $\xi$ to the point $\xi^{*}$. Then the velocity of the interface $C$ (also known as the normal velocity) is defined as

$$
C=\lim _{\tau^{*} \rightarrow \tau} \frac{\Delta \mathbf{z} \cdot \mathbf{N}}{\tau^{*}-\tau}
$$

where $\mathbf{N}$ is the unit normal to the surface $S_{\tau}$. By construction, $\Delta \mathbf{z}$ is aligned with $\mathbf{N}$, and the dot indicates that the sign of $C$ depends on the choice of the normal. Obviously $C$ is a scalar, and it is called "velocity" rather than "speed" because the normal direction is implied. 


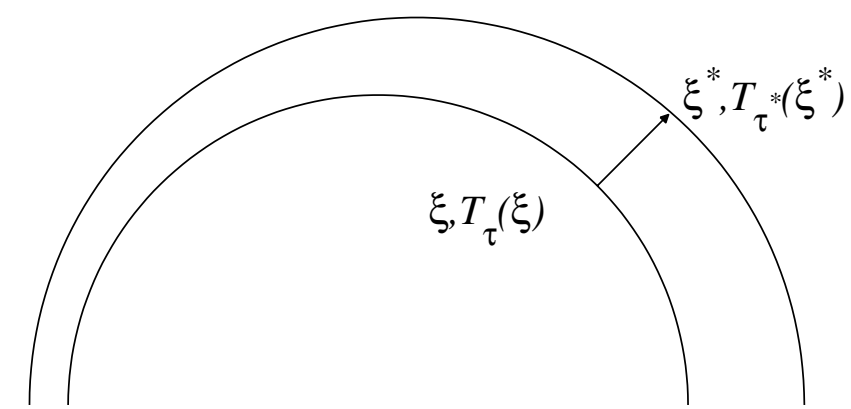

FIG. 5. Geometric definition of the $\delta / \delta \tau$-derivative as applied to a scalar field $T$ defined at time $\tau$ on the surface $S_{\tau}$.

If $\mathbf{z}$ is the radius vector with respect to an arbitrary origin, then the definition of $C$ (19) can be rewritten as

$$
C=\frac{\delta \mathbf{z}}{\delta \tau} \cdot \mathbf{N}
$$

The velocity field $C$ completely determines the evolution of the interface, much like prescribing the velocity field of a fluid completely determines its flow, with one significant difference. In the flow of a fluid, the trajectories of individual particles are usually of interest and the velocity field allows one to determine the flow. The velocity $C$, on the other hand, describes the motion of the surface as a geometric object not keeping track of individual points.

3.3. The first perturbation $\partial \psi / \partial \tau$. The perturbed bulk equation is obtained simply by differentiating Poisson's equation with respect to $\tau$ :

$$
\nabla^{i} \nabla_{i} \frac{\partial \psi}{\partial \tau}=0
$$

Since $\rho$ is constant, the would-be right-hand side $4 \pi G \frac{\partial \rho}{\partial \tau}$ vanishes. The effect of the variation in the mass density is instead captured by the boundary conditions.

Taking a $\delta / \delta \tau$-derivative (see the Appendix) of $\left[\frac{\partial \psi}{\partial \tau}\right]_{S}=0$ we get

$$
\begin{aligned}
0 & =\frac{\delta[\psi]_{S}}{\delta \tau}=\left[\frac{\delta \psi}{\delta \tau}\right]_{S}, \\
\text { by eqn. (112) } & =\left[\frac{\partial \psi}{\partial \tau}\right]_{S}+C N^{i}\left[\nabla_{i} \psi\right]_{S},
\end{aligned}
$$

where $C$ is the velocity of the interface defined above.

Since the jump in the normal derivative of $\psi$ vanishes, so does $[\partial \psi / \partial \tau]_{S}$ :

$$
\left[\frac{\partial \psi}{\partial \tau}\right]_{S}=0 .
$$


Taking the $\delta / \delta \tau$ derivative of the second boundary condition, we have

$$
\begin{aligned}
0= & \frac{\delta\left(N^{i}\left[\nabla_{i} \psi\right]_{S}\right)}{\delta \tau}=\left[\frac{\delta\left(N^{i} \nabla_{i} \psi\right)}{\delta \tau}\right]_{S}, \\
*= & \frac{\delta N^{i}}{\delta \tau}\left[\nabla_{i} \psi\right]_{S}+N^{i}\left[\frac{\delta \nabla_{i} \psi}{\delta \tau}\right]_{S}, \\
\&= & -z_{\alpha}^{i} \nabla^{\alpha} C\left[\nabla_{i} \psi\right]_{S}+N^{i}\left[\nabla_{i} \frac{\partial \psi}{\partial \tau}\right]_{S}+C N^{i} N^{j}\left[\nabla_{i} \nabla_{j} \psi\right]_{S} . \\
& { }^{*} \text { Product Rule } \\
& \text { \&By eqns. (114e) and (112) }
\end{aligned}
$$

Since $\nabla_{i} \psi$ is orthogonal to the interface at $\tau=0$, the product $z_{\alpha}^{i} \nabla_{i} \psi$ vanishes on both sides of the interface, and we have

$$
N^{i}\left[\nabla_{i} \frac{\partial \psi}{\partial \tau}\right]_{S}=-C N^{i} N^{j}\left[\nabla_{i} \nabla_{j} \psi\right]_{S} .
$$

It can be shown for a continuous function $\psi$ with continuous derivatives that

$$
\left[\nabla_{i} \nabla_{j} \psi\right]_{S}=\left(N^{k} N^{l}\left[\nabla_{k} \nabla_{l} \psi\right]_{S}\right) N_{i} N_{j}
$$

In other words, the nine entries of $\left[\nabla_{i} \nabla_{j} \psi\right]_{S}$ share a single degree of freedom (rather than six for an arbitrary function). Raising one of the indices and contracting, we get $\left[\nabla_{i} \nabla^{i} \psi\right]_{S}=4 \pi G[\rho]_{S}$. Therefore, the second boundary condition can be rewritten as

$$
N^{i}\left[\nabla_{i} \frac{\partial \psi}{\partial \tau}\right]_{S}=-4 \pi G C \rho
$$

Summarizing, the perturbed potential $\partial \psi / \partial \tau$ is governed by

$$
\begin{aligned}
\nabla^{i} \nabla_{i} \frac{\partial \psi}{\partial \tau} & =0, z \in \Omega, \\
{\left[\frac{\partial \psi}{\partial \tau}\right]_{S} } & =0, \\
N^{i}\left[\nabla_{i} \frac{\partial \psi}{\partial \tau}\right]_{S} & =-4 \pi G C \rho .
\end{aligned}
$$

Therefore, the "jump" function $J_{1}$ and the "hop" function $H_{1}$ are given by

$$
\begin{aligned}
J_{1} & =0, \\
H_{1} & =-4 \pi G R C \rho .
\end{aligned}
$$

Let us now represent $C$ as a series in spherical harmonics in a way similar to equations (15b) and (15a) except with an additional parameter $R$ for nondimensionalization:

$$
C=R C^{l m} Y_{l m}(\theta, \phi) .
$$

As $C$ is determined by the evolution $\Omega(\tau)$, so are the $C^{l m}$. We think of the $C^{l m}$ as the collection of small parameters that represent the given perturbation, and all relevant expressions are given in terms of the $C_{l m}$. Below, we shall explicitly calculate the $C^{l m}$ for two specific perturbations of the sphere: a radial perturbation that preserves the symmetry of the system and an ellipsoidal perturbation. 
Given equations (31a) and (31b), the coefficients $J_{1}^{l m}$ and $H_{1}^{l m}$ are easy to determine:

$$
\begin{aligned}
J_{1}^{l m} & =0, \\
H_{1}^{l m} & =-4 \pi G R^{2} \rho C^{l m} .
\end{aligned}
$$

We have thus obtained the first order approximation to the gravitational potential:

$$
\Delta \psi(r, \theta, \phi) \approx-\frac{4 \pi G \rho R^{2} C^{l m}}{2 l+1}\left\{\begin{array}{rl}
\left(\frac{r}{R}\right)^{l}, & z<\Omega, \\
\left(\frac{r}{R}\right)^{-l-1}, & z>\Omega,
\end{array} \quad Y_{l m}(\theta, \phi) .\right.
$$

4. Second and third perturbations. The system of equations for $\partial^{2} \psi / \partial \tau^{2}$ is obtained by repeating the steps of the previous section, i.e. by differentiating equations (28)-30 we respect to $\tau$ :

$$
\begin{aligned}
\nabla^{i} \nabla_{i} \frac{\partial^{2} \psi}{\partial \tau^{2}} & =0, z \in \Omega, \\
{\left[\frac{\partial^{2} \psi}{\partial \tau^{2}}\right]_{S} } & =4 \pi G \rho C^{2}, \\
N^{i}\left[\nabla_{i} \frac{\partial^{2} \psi}{\partial \tau^{2}}\right]_{S} & =-4 \pi G \rho \frac{\delta C}{\delta \tau}+4 \pi G \rho C^{2} B_{\alpha}^{\alpha},
\end{aligned}
$$

where $B_{a}^{\alpha}$ is the mean curvature (102) which, for a sphere of radius $R$, equals $-2 / R$. We rewrite the last boundary condition as

$$
N^{i}\left[\nabla_{i} \frac{\partial^{2} \psi}{\partial \tau^{2}}\right]_{S}=-4 \pi G \rho\left(\frac{2 C^{2}}{R}+\frac{\delta C}{\delta \tau}\right) .
$$

$J_{2}$ and $H_{2}$ are therefore given by

$$
\begin{aligned}
J_{2} & =4 \pi G \rho C^{2} \\
H_{2} & =-4 \pi G \rho\left(2 C^{2}+R \frac{\delta C}{\delta \tau}\right) .
\end{aligned}
$$

The surface fields $J_{2}$ and $H_{2}$ cannot be as easily decomposed in spherical harmonics as $J_{1}$ (which was zero) and $H_{1}$ (which was directly proportional to $C$ ). Therefore, for a given perturbation they need to be explicitly computed and independently expressed in spherical harmonics. Below we perform this task for radial and ellipsoidal perturbations.

Finally, we present the system for $\partial^{3} \psi / \partial \tau^{3}$, which is obtained analogously to $\partial \psi / \partial \tau$ and $\partial^{2} \psi / \partial \tau^{2}$ :

$$
\begin{aligned}
\nabla^{i} \nabla_{i} \frac{\partial^{2} \psi}{\partial \tau^{2}} & =0, z \in \Omega, \\
{\left[\frac{\partial^{2} \psi}{\partial \tau^{2}}\right]_{S} } & =4 \pi G \rho\left(3 C \frac{\delta C}{\delta \tau}-C^{3} B_{\alpha}^{\alpha}\right), \\
N^{i}\left[\nabla_{i} \frac{\partial^{2} \psi}{\partial \tau^{2}}\right]_{S} & =-4 \pi G \rho\left(\begin{array}{c}
\frac{\delta^{2} C}{\delta \tau^{2}}-3 C \frac{\delta C}{\delta \tau} B_{\alpha}^{\alpha}-3 C^{2} \nabla^{\alpha} \nabla_{\alpha} C-\ldots \\
\ldots-4 C \nabla^{\alpha} C \nabla_{\alpha} C-C^{3} B_{\beta}^{\alpha} B_{\alpha}^{\beta}+C^{3} B_{\alpha}^{\alpha} B_{\beta}^{\beta}
\end{array}\right),
\end{aligned}
$$


where (having substituted $B_{\alpha}^{\alpha}=-2 / R$ and $B_{\beta}^{\alpha} B_{a}^{\beta}=2 / R^{2}$ )

$$
\begin{aligned}
J_{3} & =4 \pi G \rho\left(3 C \frac{\delta C}{\delta \tau}+\frac{2 C^{3}}{R}\right) \\
H_{3} & =-4 \pi G \rho\left(\begin{array}{c}
R \frac{\delta^{2} C}{\delta \tau^{2}}+6 C \frac{\delta C}{\delta \tau}-3 R C^{2} \nabla^{\alpha} \nabla_{\alpha} C-\ldots \\
\ldots-4 R C \nabla^{\alpha} C \nabla_{\alpha} C+\frac{2 C^{3}}{R}
\end{array}\right) .
\end{aligned}
$$

We reiterate that the perturbation equations are started only in terms of the operator $\delta / \delta \tau$ and the velocity field $C$ along with the stationary characteristics of the surface such as the mean curvature $B_{\alpha}^{\alpha}$ and the trace of the third groundform $B_{\beta}^{\alpha} B_{\alpha}^{\beta}$.

\section{Two examples.}

5.1. Spherical expansion. We consider a spherical configuration with radius $R(1+\varepsilon a)$, where $\varepsilon$ is a small quantity and $a$ is on the order of unity. The gravitational potential for this geometry can be obtained directly, and in fact it is available in equation (12):

$$
\psi_{0}(\varepsilon, r)=\frac{2 \pi}{3} G \rho\left(r^{2}-3 R^{2}(1+\varepsilon a)^{2}\right) .
$$

Therefore, the perturbation $\Delta \psi=\psi_{0}(\varepsilon, r)-\psi_{0}(r)$ is given by

$$
\Delta \psi=2 \pi G \rho R^{2}\left(2 \varepsilon a+\varepsilon^{2} a^{2}\right) .
$$

This expression is a polynomial in $\varepsilon$, so we expect that our two-term Taylor series approximation will recover the correction exactly.

As prescribed by the recipe in the Appendix we specify an evolution of the initial configuration into the perturbed one. We let the radius evolve according to $R(1+\tau \varepsilon a)$. The velocity of the interface for this evolution is given by

$$
C(\theta, \phi)=R \varepsilon a,
$$

and their spherical harmonic expansions have the single $l=0, m=0$ term:

$$
C^{00}=\sqrt{4 \pi} \varepsilon a .
$$

Therefore, we have

$$
J_{1}^{00}=0 ; \quad H_{1}^{00}=\sqrt{4 \pi} \varepsilon a .
$$

Moving on to the second order perturbation, we have

$$
\begin{aligned}
J_{2} & =-\frac{C^{2}}{R^{2}}=-\varepsilon^{2} a^{2}, \\
H_{2} & =\frac{2 C^{2}}{R^{2}}+\frac{1}{R} \frac{\delta C}{\delta \tau}=2 \varepsilon^{2} a^{2},
\end{aligned}
$$

since it is clear that $\delta C / \delta \tau$ vanishes

$$
\begin{aligned}
J_{2}^{00} & =-\sqrt{4 \pi} \varepsilon^{2} a^{2}, \\
H_{2}^{00} & =2 \sqrt{4 \pi} \varepsilon^{2} a .
\end{aligned}
$$

Constructing the Taylor series inside the domain, we get

$$
\Delta \psi \approx-2 \pi G \rho R^{2}\left(2 \varepsilon a+\varepsilon^{2} a^{2}\right),
$$

which is precisely equation (42). 
5.2. Ellipsoidal perturbation. We now turn to the computation of the potential inside a near-spherical ellipsoidal domain. The potential outside this domain can be computed analogously, but the results are not presented here for consideration of space.

The gravitational potential of a near-spherical ellipsoid can be estimated to any order by a technique originally proposed by Migdal [8, [5] for the analysis of the energy splitting of a quantum particle trapped in an ellipsoidal cavity. Migdal's technique is simpler than ours (although not shorter!) for lower order perturbations and can be used for an independent verification of our results.

The gravitational correction inside a slightly ellipsoidal shell was also calculated to second order in 10 .

The boundary $S$ is an ellipsoid with semi-axes $R(1+\varepsilon a), R(1+\varepsilon b), R(1+\varepsilon c)$. As before, we start by specifying the evolution from $\Omega_{0}$ (a sphere of radius $R$ ) to $\Omega_{1}$ (our ellipsoid):

$$
\left[\begin{array}{l}
z^{1} \\
z^{2} \\
z^{3}
\end{array}\right]=R\left[\begin{array}{l}
(1+\tau a) \sin \theta \cos \phi \\
(1+\tau b) \sin \theta \sin \phi \\
(1+\tau c) \cos \theta
\end{array}\right] .
$$

5.2.1. Computing $C$. The marker velocity $v^{i}$ (107a) is given by

$$
v^{i}=\frac{\partial z^{i}}{\partial \tau}=R\left[\begin{array}{l}
a \sin \theta \cos \phi \\
b \sin \theta \sin \phi \\
c \cos \theta
\end{array}\right],
$$

and the velocity $C$ of the boundary at $\tau=0$ is constructed by projecting the marker velocity onto the unperturbed normal $N^{i}=(a \sin \theta \cos \phi, b \sin \theta \sin \phi, c \cos \theta)$ :

$$
\left.C\right|_{\tau=0}=R\left(a \sin ^{2} \theta \cos ^{2} \phi+b \sin ^{2} \theta \sin ^{2} \phi+c \cos ^{2} \theta\right) .
$$

This expression is easy to represent in terms of $Y_{l m}$ as in equation (32), since only a few terms are nonzero:

$$
\begin{aligned}
C^{00}= & \sqrt{\frac{4 \pi}{9}}(a+b+c), \\
C^{2 m}= & {\left[\begin{array}{c}
\sqrt{\frac{2 \pi}{15}}(a-b) \\
0 \\
-\sqrt{\frac{4 \pi}{45}}(a+b-2 c) \\
0 \\
\sqrt{\frac{2 \pi}{15}}(a-b)
\end{array}\right] . }
\end{aligned}
$$

These coefficients can practically be obtained from equation (52) in a number of ways. One is by integration:

$$
C^{l m}=\frac{1}{R} \int_{-\pi}^{\pi} \int_{0}^{\pi} C(\theta, \phi) Y_{l m}^{*}(\theta, \phi) \sin \theta d \theta d \phi,
$$

where $*$ stands for complex conjugation. 
Alternatively, one can expand the harmonic series for $C$ (32) as a single trigonometric polynomial and form a linear system for $C^{l m}$ by matching up the terms of the trigonometric polynomials with the explicit expression (52) for $C$.

5.3. First order coefficients. Decomposing $J_{1}=0$ and $H_{1}=C / R$, defined by equations (14a), 114b), with respect to spherical harmonics yields the following coefficients (15a), (15b):

$$
\begin{aligned}
& \left.\frac{(l+1) J_{1}^{l m}+H_{1}^{l m}}{-4 \pi G R^{2} \rho}\right|_{l=0}=\left.\frac{-l J_{1}^{l m}+H_{1}^{l m}}{-4 \pi G R^{2} \rho}\right|_{l=0}=\sqrt{\frac{4 \pi}{9}(a+b+c),} \\
& \left.\frac{(l+1) J_{1}^{l m}+H_{1}^{l m}}{-4 \pi G R^{2} \rho}\right|_{l=2}=\left.\frac{-l J_{1}^{l m}+H_{1}^{l m}}{-4 \pi G R^{2} \rho}\right|_{l=2}=\left[\begin{array}{c}
\sqrt{\frac{2 \pi}{15}}(a-b) \\
0 \\
-\sqrt{\frac{4 \pi}{45}}(a+b-2 c) \\
0 \\
\sqrt{\frac{2 \pi}{15}}(a-b)
\end{array}\right] .
\end{aligned}
$$

5.3.1. Computing $\delta C / \delta \tau$. According to the definition of the $\delta / \delta \tau$-derivative (108), we have

$$
\frac{\delta C}{\delta \tau}=\frac{\partial C(\tau, \theta, \phi)}{\partial \tau}-v^{i} z_{i}^{\alpha} \nabla_{\alpha} C
$$

The term $\partial C / \partial \tau$ contains a partial derivative of the normal $N^{i}$ with respect to $\tau$. This would be a very cumbersome computation, given the definition of the normal in equation (95). It can be avoided by utilizing equations (111) and (114e) to eliminate the $\partial N^{i} / \partial \tau$ term. The resulting expression is

$$
\frac{\delta C}{\delta \tau}=-v_{i} z_{\alpha}^{i}\left(N_{k} \nabla^{\alpha} v^{k}+\nabla^{\alpha} C\right) .
$$

The shift tensor $z_{\alpha}^{i}$ (87) is given by

$$
z_{\alpha}^{i}=R\left[\begin{array}{cc}
\cos \theta \cos \phi & -\sin \theta \sin \phi \\
\cos \theta \sin \phi & \sin \theta \cos \phi \\
-\sin \theta & 0
\end{array}\right] .
$$

Since $C$ is a scalar, the surface gradient (97) of $C$ coincides with the ordinary gradient:

$$
\nabla_{\alpha} C=R\left[\begin{array}{c}
\left(\frac{1}{2} a-\frac{1}{2} b\right) \sin 2 \theta \cos 2 \phi-\left(c-\frac{1}{2} a-\frac{1}{2} b\right) \sin 2 \theta \\
-(a-b) \sin ^{2} \theta \sin 2 \phi
\end{array}\right]
$$

The surface gradient of the marker velocity coincides with the ordinary gradient, since a Cartesian metric is used in the embedding space:

$$
\nabla_{\alpha} v^{i}=R\left[\begin{array}{rr}
\cos \theta \cos \phi & -\sin \theta \sin \phi \\
\cos \theta \sin \phi & \sin \theta \cos \phi \\
-\sin \theta & 0
\end{array}\right]
$$


Putting everything together we obtain the following expression:

$$
\begin{gathered}
\frac{\delta C}{\delta \tau}=-\left[\begin{array}{l}
a \sin \theta \cos \phi \\
b \sin \theta \sin \phi \\
c \cos \theta
\end{array}\right]^{T}\left[\begin{array}{rr}
\cos \theta \cos \phi & -\sin \theta \sin \phi \\
\cos \theta \sin \phi & \sin \theta \cos \phi \\
-\sin \theta & 0
\end{array}\right]\left[\begin{array}{ll}
1 & 0 \\
0 & \sin ^{2} \theta
\end{array}\right]^{-1}\left[\begin{array}{l}
\frac{\partial C}{\partial \theta} \\
\frac{\partial C}{\partial \phi}
\end{array}\right] \\
-\left[\begin{array}{l}
a \sin \theta \cos \phi \\
b \sin \theta \sin \phi \\
c \cos \theta
\end{array}\right]^{T}\left[\begin{array}{rr}
\cos \theta \cos \phi & -\sin \theta \sin \phi \\
\cos \theta \sin \phi & \sin \theta \cos \phi \\
-\sin \theta & 0
\end{array}\right]\left[\begin{array}{ll}
1 & 0 \\
0 & \sin ^{2} \theta
\end{array}\right] \\
\times\left[\begin{array}{rr}
a \cos \theta \cos \phi & -a \sin \theta \sin \phi \\
b \cos \theta \sin \phi & b \sin \theta \cos \phi \\
-c \sin \theta & 0
\end{array}\right]^{T}\left[\begin{array}{l}
\sin \theta \cos \phi \\
\sin \theta \sin \phi \\
\cos \theta
\end{array}\right] .
\end{gathered}
$$

5.3.2. Second order coefficients. The following equations summarize the values for $(l+1) J_{n}^{l m}+H_{n}^{l m}$ and $-l J_{n}^{l m}+H_{n}^{l m}$, which were obtained using Mathematica [13:

$$
\begin{aligned}
& \left.\frac{(l+1) J_{2}^{l m}+H_{2}^{l m}}{4 \pi G R^{2} \rho}\right|_{l=0}=\frac{2}{15} \sqrt{\pi}\left(3 a^{2}+3 b^{2}+3 c^{2}-8 a b-8 b c-8 c a\right), \\
& \left.\frac{(l+1) J_{2}^{l m}+H_{2}^{l m}}{4 \pi G R^{2} \rho}\right|_{l=2}=\left[\begin{array}{c}
\frac{\sqrt{30 \pi}}{105}(a-b)(9 a+9 b-4 c) \\
0 \\
-\frac{2 \sqrt{5 \pi}}{105}\left(9 a^{2}+9 b^{2}-18 c^{2}-8 a b+4 b c+4 c a\right) \\
0 \\
\frac{\sqrt{30 \pi}}{105}(a-b)(9 a+9 b-4 c)
\end{array}\right] \text {, } \\
& \left.\frac{-l J_{2}^{l m}+H_{2}^{l m}}{4 \pi G R^{2} \rho}\right|_{l=0}=-\frac{4 \sqrt{\pi}}{3}(a b+b c+c a) \text {, } \\
& \left.\frac{-l J_{2}^{l m}+H_{2}^{l m}}{-4 \pi G R^{2} \rho}\right|_{l=2}=\left[\begin{array}{c}
\frac{\sqrt{30 \pi}}{15}(a-b)(3 a+3 b+2 c) \\
0 \\
-\frac{2 \sqrt{5 \pi}}{15}\left(3 a^{2}+3 b^{2}-6 c^{2}+4 a b-2 b c-2 c a\right) \\
0 \\
\frac{\sqrt{30 \pi}}{15}(a-b)(3 a+3 b+2 c)
\end{array}\right], \\
& -\frac{3 \sqrt{70 \pi}}{35}(a-b)^{2} \\
& 0 \\
& \frac{6 \sqrt{10 \pi}}{35}(a-b)(a+b-2 c) \\
& \left.\frac{-l J_{2}^{l m}+H_{2}^{l m}}{4 \pi G R^{2} \rho}\right|_{l=4}=\left[\begin{array}{c}
0 \\
-\frac{6 \sqrt{\pi}}{35}\left(3 a^{2}+3 b^{2}+8 c^{2}+2 a b-8 b c-8 c a\right) \\
0 \\
\frac{6 \sqrt{10 \pi}}{35}(a-b)(a+b-2 c) \\
0 \\
-\frac{3 \sqrt{70 \pi}}{35}(a-b)^{2}
\end{array}\right] .
\end{aligned}
$$

Interestingly, according to equation (62b) there is no contribution from the $l=4$ harmonics inside the ellipsoid, consistent with the fact that inside the ellipsoid with arbitrary semi-axes the potential is a linear function of $x^{2}, y^{2}$, and $z^{2}$, each of which can be represented as a linear combination of $r^{2} Y_{2 m}$. 
5.3.3. Third order coefficients. Finally, we present the coefficients for the third order perturbation:

$$
\begin{aligned}
\left.\frac{(l+1) J_{3}^{l m}+H_{3}^{l m}}{4 \pi G R^{2} \rho}\right|_{l=0}= & -\frac{8 \sqrt{\pi}}{35}(a+b-2 c)(2 a-b-c)(a-2 b+c), \\
\left.\frac{(l+1) J_{3}^{l m}+H_{3}^{l m}}{4 \pi G R^{2} \rho}\right|_{l=2}= & {\left[\begin{array}{c}
-\frac{2}{7} \sqrt{\frac{2 \pi}{15}}(a-b)\left(\begin{array}{c}
8 a^{2}+8 b^{2}-5 c^{2} \\
+12 a b-b c-a c \\
0
\end{array}\right) \\
\frac{4}{21} \sqrt{\frac{\pi}{5}}\left(\begin{array}{c}
8 a^{3}+8 b^{3}-16 c^{3} \\
-6 a^{2} b-6 a b^{2}-3 a c^{2}-3 b c^{2} \\
+9 b^{2} c+9 a^{2} c \\
0 \\
8 a^{2}+8 b^{2}-5 c^{2} \\
+12 a b-b c-a c
\end{array}\right)
\end{array}\right], }
\end{aligned}
$$

$$
\begin{aligned}
& \left.\frac{-l J_{3}^{l m}+H_{3}^{l m}}{4 \pi G R^{2} \rho}\right|_{l=0}=4 \sqrt{\pi} a b c,
\end{aligned}
$$

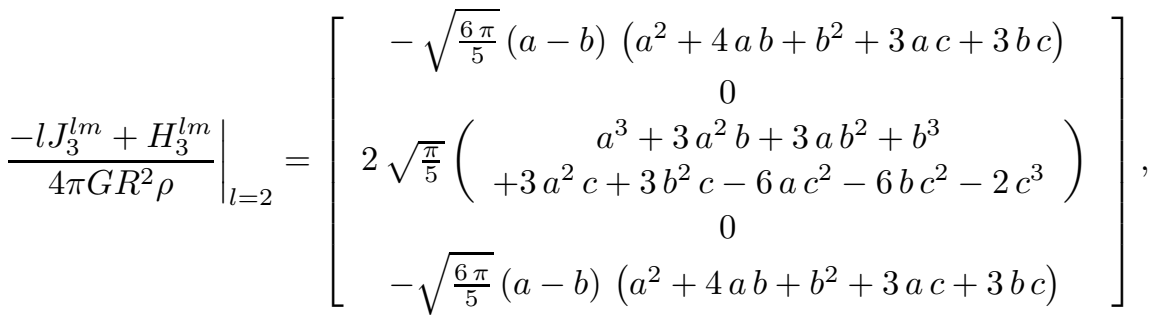

$$
\begin{aligned}
& -9(a-b)^{2}(2 a+2 b+c) \sqrt{\frac{2 \pi}{35}} \\
& 0 \\
& \frac{18}{7} \sqrt{\frac{2 \pi}{5}}(a-b)\left(\begin{array}{c}
2 a^{2}+2 b^{2}-3 c^{2} \\
+3 a b-2 a c-2 b c
\end{array}\right) \\
& 0 \\
& \left.\frac{-l J_{3}^{l m}+H_{3}^{l m}}{4 \pi G R^{2} \rho}\right|_{l=4}=-\frac{18}{35} \sqrt{\pi}\left(\begin{array}{c}
6 a^{3}+6 b^{3}+16 c^{3}+6 a^{2} b+6 a b^{2} \\
-9 a^{2} c-9 b^{2} c-4 a c^{2}-4 b c^{2} \\
-14 a b c \\
0 \\
\frac{18}{7} \sqrt{\frac{2 \pi}{5}}(a-b)\left(\begin{array}{c}
2 a^{2}+2 b^{2}-3 c^{2} \\
+3 a b-2 a c-2 b c
\end{array}\right)
\end{array}\right. \\
& 0 \\
& -9 \sqrt{\frac{2 \pi}{35}}(a-b)^{2}(2 a+2 b+c)
\end{aligned}
$$




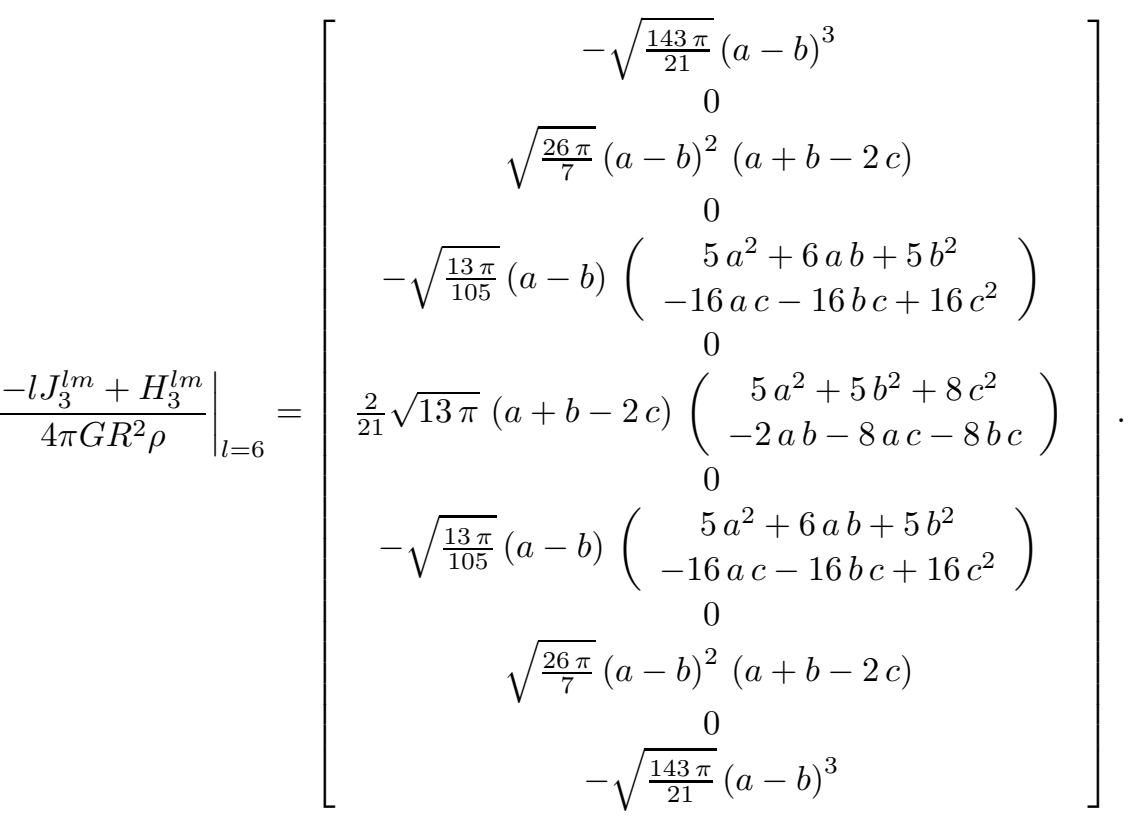

5.3.4. Analysis of accuracy. We considered an ellipsoid of density $\rho=1$ with semiaxes $2(1+\varepsilon), 2(1+2 \varepsilon), 2(1+3 \varepsilon)$, i.e. $R=2, a=1, b=2, c=3$. The potential inside a uniform ellipsoid with semi-axes $A, B$, and $C$ is given in closed form by the elliptical integral

$$
\psi(x, y, z)=\pi G \rho A B C \int_{0}^{\infty} \frac{1-\frac{x^{2}}{A^{2}+q}-\frac{y^{2}}{B^{2}+q}-\frac{z^{2}}{C^{2}+q}}{\sqrt{\left(A^{2}+q\right)\left(B^{2}+q\right)\left(C^{2}+q\right)}} d q,
$$

which can be evaluated to arbitrary precision (we used 40 digits) by a number of software packages. We selected a single point $(r=1 / 2, \theta=\pi / 3, \phi=\pi / 3)$ and compared the "true" value obtained from the integral above to the Taylor series estimates for $\varepsilon=10^{-1}$, $10^{-2}, 10^{-3}, 10^{-4}, 10^{-5}$, and $10^{-6}$. Figure 6 shows the error with the Taylor series estimates for zeroth, first, second, and third order estimates. Observe that the slopes of the curves are respectively $-1,-2,-3$, and -4 , indicating that the produced errors behave as $O\left(\varepsilon^{n+1}\right)$, where $n$ is the order of the Taylor estimate.

In the table below we present the true values and our best Taylor series estimates for the potential at $(r=1 / 2, \theta=\pi / 3, \phi=\pi / 3)$ :

$$
\begin{aligned}
& \varepsilon \quad \text { True value to } 40 \text { digits } \quad \text { Taylor estimate |Error| } \\
& \begin{array}{llll}
10^{-1}-35.43708351803048697192025381012391736957 & \frac{-18950357 \pi}{1680000} & 1.20 \times 10^{-7}
\end{array} \\
& 10^{-2}-25.62255732018668419194698852443368280613 \quad \frac{-856370897 \pi}{10500000} \quad 1.37 \times 10^{-9} \\
& 10^{-3}-24.70978981647731607371769060209184738688 \quad \frac{-943844446093 \pi}{120000000000} \quad 1.54 \times 10^{-13} \\
& 10^{-4}-24.61920024789102747620130556538189421147 \quad \frac{-6582689256227401 \pi}{84000000000000} \quad 1.56 \times 10^{-17} \\
& 10^{-5}-24.61014816318116756083082018753515760828 \quad \frac{-6580268907062274901 \pi}{8400000000000000} \quad 1.56 \times 10^{-21} \\
& 10^{-6}-24.60924302343199898214889695992182000437 \frac{-6580026890520622749901 \pi}{84000000000000000000} 1.56 \times 10^{-25}
\end{aligned}
$$




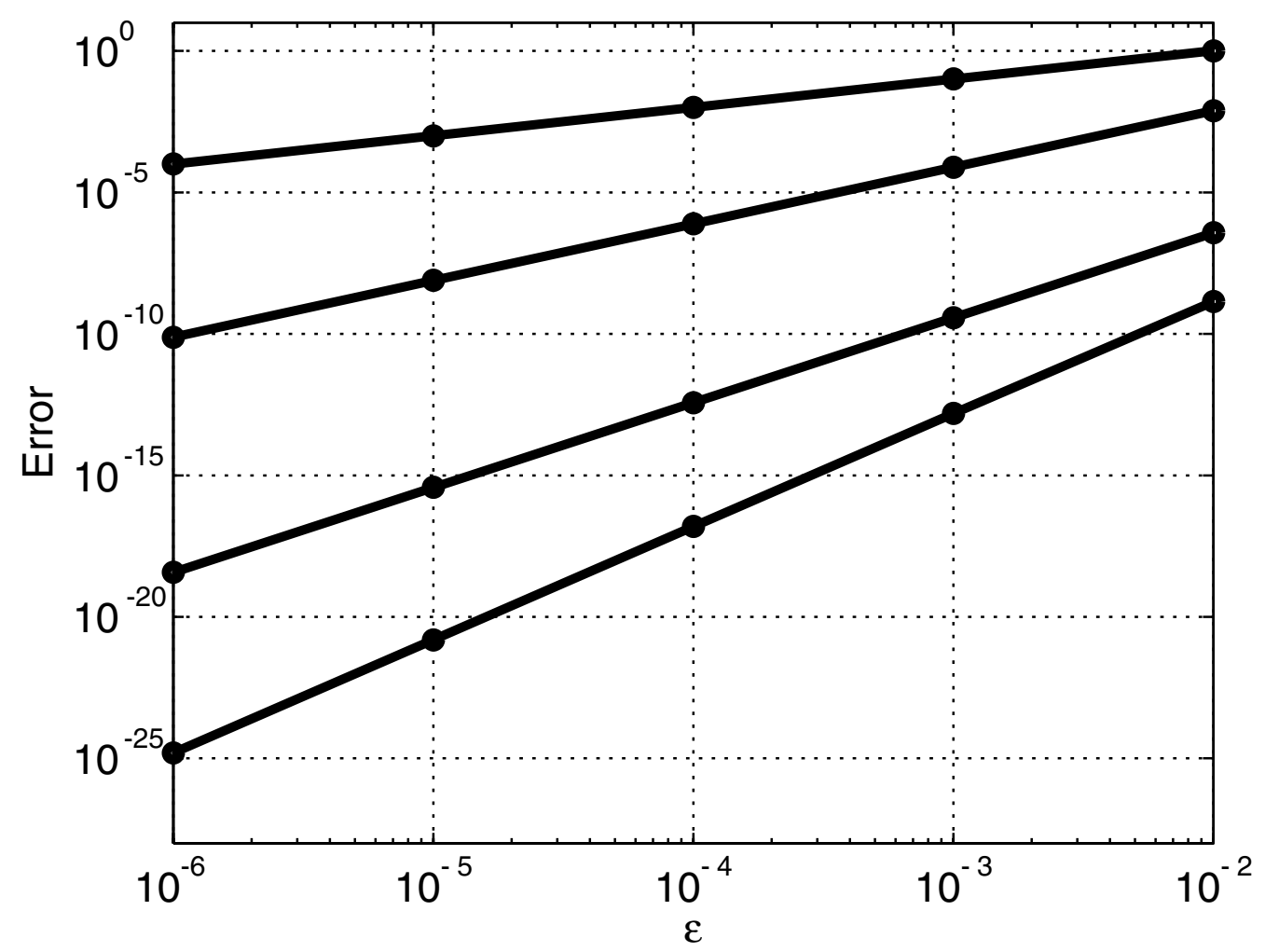

FIG. 6. The error in the Taylor series estimate for $\varepsilon=10^{-2}, 10^{-3}$, $10^{-4}, 10^{-5}, 10^{-6}$. The four curves correspond to the Taylor series with zero, one, two, and three terms.

The ellipticity of the Earth is roughly on the order of $10^{-2}$. Since the density of the Earth varies with distance from the center, our technique is not directly applicable. However, an adaptation of our technique would be applicable, and Figure 6 indicates that the second order estimate will produce a relative error on the order of $5 \times 10^{-11}$.

6. First order perturbation for a triaxial planet. This section is concerned with an example that has a number of geophysical applications related to the dynamics of the inner core and also shows how to apply the obtained expressions to composite geometries. Suppose that the configuration (before the perturbation) consists of an inner core $\Omega_{1}$ of radius $R_{1}$ and density $\rho_{1}$, an outer core $\Omega_{2}$ of radius $R_{2}$ and density $\rho_{2}$, and a mantle $\Omega_{3}$ of radius $R_{3}$ and density $\rho_{3}$. This configuration can be equivalently represented by three superimposed spheres: one of radius $R_{3}$ and density $\rho_{3}$, another of radius $R_{2}$ and density $\rho_{2}-\rho_{3}$, and a third one of radius $R_{1}$ and density $\rho_{1}-\rho_{2}$.

We construct the estimates for the resulting potential by adjusting expressions for the unperturbed potential (12) and the first order perturbation (34) to each of the domains and then combining them. In order to make equations (12) and (34) appear more generic, introduce a radius $R_{*}$ and a density $\rho_{*}$. Also, define the dimensionless densities $\varrho_{n}$ and 
radii $Q_{n}$ :

$$
\varrho_{n}=\frac{\rho_{n}}{\rho_{*}}, \quad Q_{n}=\frac{R_{n}}{R_{*}},
$$

and a convenient quantity $\Psi_{*}$ that has dimensions of gravitational potential:

$$
\Psi_{*}=\frac{4 \pi}{3} G \rho_{*} R_{*}^{2} .
$$

Consistent with the definition (10), let $[\varrho]_{1}=\varrho_{1}-\varrho_{2},[\varrho]_{2}=\varrho_{2}-\varrho_{3}$, and $[\varrho]_{3}=\varrho_{3}$.

6.1. The unperturbed potential. The unperturbed potential for a single domain (say $\Omega_{1}$ ), equation (12), can now be rewritten as

$$
\psi_{0}(r)=\Psi_{*}\left\{\begin{aligned}
\frac{[\varrho]_{1}}{2}\left(\frac{r}{R_{*}}\right)^{2}-\frac{3}{2}[\varrho]_{1} Q_{1}^{2}, & z<\Omega_{1}, \\
-[\varrho]_{1} Q_{1}^{3}\left(\frac{r}{R_{*}}\right)^{-1}, & z>\Omega_{1},
\end{aligned}\right.
$$

and the combined expression is easily obtained by replacing the subscript of 1 with 2 and 3:

$$
\psi_{0}(r)=\Psi_{*}\left\{\begin{array}{rr}
\frac{[\varrho]_{1}}{2}\left(\frac{r}{R_{*}}\right)^{2}-\frac{3}{2}[\varrho]_{1} Q_{1}^{2}, & z<\Omega_{1}, \\
-[\varrho]_{1} Q_{1}^{3}\left(\frac{r}{R_{*}}\right)^{-1}, & z>\Omega_{1}, \\
+\frac{[\varrho]_{2}}{2}\left(\frac{r}{R_{*}}\right)^{2}-\frac{3}{2}[\varrho]_{2} Q_{2}^{2}, & z<\Omega_{2}, \\
-[\varrho]_{2} Q_{2}^{3}\left(\frac{r}{R_{*}}\right)^{-1}, & z>\Omega_{2}, \\
+\frac{[\varrho]_{3}}{2}\left(\frac{r}{R_{*}}\right)^{2}-\frac{3}{2}[\varrho]_{3} Q_{3}^{2}, & z<\Omega_{3}, \\
-[\varrho]_{3} Q_{3}^{3}\left(\frac{r}{R_{*}}\right)^{-1}, & z>\Omega_{3} .
\end{array}\right.
$$

Rewriting the sum in a single expression, we get

$$
\psi_{0}(r)=\Psi_{*} \begin{cases}\frac{\varrho_{1}}{2}\left(\frac{r}{R_{*}}\right)^{2}+A_{1}, & z<\Omega_{1}, \\ \frac{\varrho_{2}}{2}\left(\frac{r}{R_{*}}\right)^{2}+A_{2}+B_{2}\left(\frac{r}{R_{*}}\right)^{-1}, & \Omega_{1}<z<\Omega_{2}, \\ \frac{\varrho_{3}}{2}\left(\frac{r}{R_{*}}\right)^{2}+A_{3}+B_{3}\left(\frac{r}{R_{*}}\right)^{-1}, & \Omega_{2}<z<\Omega_{3}, \\ B_{4}\left(\frac{r}{R_{*}}\right)^{-1}, & \Omega_{3}<z,\end{cases}
$$

where

$$
\left[\begin{array}{c}
A_{1} \\
A_{2} \\
A_{3} \\
B_{2} \\
B_{3} \\
B_{4}
\end{array}\right]=\left[\begin{array}{r}
-\frac{3}{2}[\varrho]_{1} Q_{1}^{2}-\frac{3}{2}[\varrho]_{2} Q_{2}^{2}-\frac{3}{2}[\varrho]_{3} Q_{3}^{2} \\
-\frac{3}{2}[\varrho]_{2} Q_{2}^{2}-\frac{3}{2}[\varrho]_{3} Q_{3}^{2} \\
-\frac{3}{2}[\varrho]_{3} Q_{3}^{2} \\
-[\varrho]_{1} Q_{1}^{3} \\
-[\varrho]_{1} Q_{1}^{3}-[\varrho]_{2} Q_{2}^{3} \\
-[\varrho]_{1} Q_{1}^{3}-[\varrho]_{2} Q_{2}^{3}-[\varrho]_{3} Q_{3}^{3}
\end{array}\right]
$$


6.2. The first order perturbation. We assume that each of the three domains $\Omega_{n}$ is perturbed in its own way according to $C_{n}$ and that each $C_{n}$ is represented as a series in spherical harmonics

$$
C_{n}=R_{n} C_{n}^{l m} Y_{l m}
$$

We first rewrite the first order perturbation for a single domain (say $\Omega_{1}$ ) as

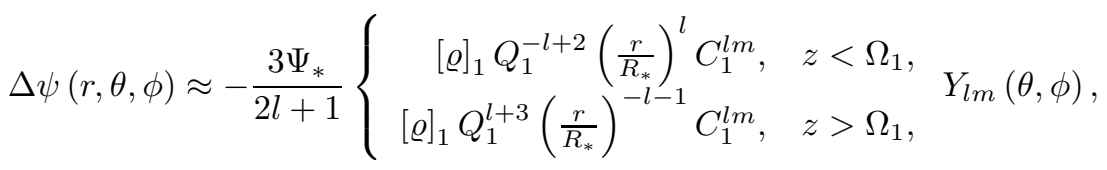

and the combined expression can be obtained similarly to the unperturbed potential:

$$
\frac{\partial \psi}{\partial \tau}(r, \theta, \phi)=\Psi_{*}\left\{\begin{array}{rr}
A_{1}^{l m}\left(\frac{r}{R_{*}}\right)^{l}, & z<\Omega_{1} \\
A_{2}^{l m}\left(\frac{r}{R_{*}}\right)^{l}+B_{2}^{l m}\left(\frac{r}{R_{*}}\right)^{-l-1}, & \Omega_{1}<z<\Omega_{2} \\
A_{3}^{l m}\left(\frac{r}{R_{*}}\right)^{l}+B_{3}^{l m}\left(\frac{r}{R_{*}}\right)^{-l-1}, & \Omega_{2}<z<\Omega_{3} \\
B_{4}^{l m}\left(\frac{r}{R_{*}}\right)^{-l-1}, & \Omega_{3}<z
\end{array} \mid Y_{l m}(\theta, \phi),\right.
$$

where

$$
\left[\begin{array}{c}
A_{1}^{l m} \\
A_{2}^{l m} \\
A_{3}^{l m} \\
B_{2}^{l m} \\
B_{3}^{l m} \\
B_{4}^{l m}
\end{array}\right]=-\frac{3}{2 l+1}\left[\begin{array}{ccc}
Q_{1}^{-l+2} & Q_{2}^{-l+2} & Q_{3}^{-l+2} \\
0 & Q_{2}^{-l+2} & Q_{3}^{-l+2} \\
0 & 0 & Q_{3}^{-l+2} \\
Q_{1}^{l+3} & 0 & 0 \\
Q_{1}^{l+3} & Q_{2}^{l+3} & 0 \\
Q_{1}^{l+3} & Q_{2}^{l+3} & Q_{3}^{l+3}
\end{array}\right]\left[\begin{array}{l}
{[\varrho]_{1} C_{1}^{l m}} \\
{[\varrho]_{2} C_{2}^{l m}} \\
{[\varrho]_{3} C_{3}^{l m}}
\end{array}\right]
$$

We have computed a first order correction to the graviational potential of a piecewise constant density tri-layer planet for aribtrary small deformations of the three interfaces. Such deformations are typically caused by the planet's rotation (Earth) or by thermal convection in the interior of the planet (Mercury). In both cases, the deformations may be treated as ellipsoidal for a number of purposes. Therefore, the resulting corrections to the gravitational field may be obtained by substituting the ellipsoidal expansion coefficients $C^{l m}$, equations (53a)-(53b), into the expressions for $\partial \psi / \partial \tau$, equations (71)-(72). The resulting estimates are consistent with the estimates obtained by Szeto and $\mathrm{Xu}$ in 10 . for the gravitational potential inside an ellipsoidal cavity.

\section{Appendix: A concise exposition of the $\delta / \delta \tau$-derivative.}

7.1. Objects defined in a Euclidean space. The radius vector $\mathbf{z}$ extends from an arbitrarily selected pole $O$ to points in the Euclidean space. In a coordinate system $z^{i}$ (i.e. $\left.\left(z^{1}, z^{2}, z^{3}\right)\right)$, the radius vector $\mathbf{z}$ becomes a (vector-valued) function of $z^{i}: \mathbf{z}=\mathbf{z}\left(z^{i}\right)$. This definition overloads the symbol $\mathbf{z}$ - it now stands for both an invariant vector field and its functional representation. We almost always omit the indices in the arguments of a function and simply write

$$
\mathbf{z}=\mathbf{z}(z)
$$

implying $\mathbf{z}=\mathbf{z}\left(z^{1}, z^{2}, z^{3}\right)$. 
The covariant basis $\mathbf{z}_{i}$ is defined at every point in space as

$$
\mathbf{z}_{i}=\frac{\partial \mathbf{z}}{\partial z^{i}}
$$

The covariant basis is a (vector-valued) tensor which can be shown by a simple application of the chain rule.

The covariant metric tensor $z_{i j}$ is defined as

$$
z_{i j}=\mathbf{z}_{i} \cdot \mathbf{z}_{j}
$$

Consistent with its name, it is a doubly covariant tensor which follows from the fact that the dot product is a tensor operation. As a matrix it is symmetric and positive (semi-)definite.

When it leads to no confusion, the determinant $\left|z_{i j}\right|$ (not a tensor) is denoted by $z$.

The permutation tensors $z_{i j k}$ and $z^{i j k}$ are defined as

$$
z_{i j k}=\sqrt{z} e^{i j k}, \quad z^{i j k}=\frac{1}{\sqrt{z}} e^{i j k}
$$

where

$$
e^{i j k}, e_{i j k}=\left\{\begin{array}{l}
+1, \text { if } i, j, k \text { is a positive permutation, } \\
-1, \text { if } i, j, k \text { is a negative permutation } \\
0, \text { otherwise. }
\end{array}\right.
$$

The contravariant metric tensor $z^{i j}$ is defined as the matrix inverse of $z_{i j}$ :

$$
z_{i j} z^{j k}=\delta_{i}^{k} \text {. }
$$

Linear algebra dictates that contraction by the other index ("commuting" in the language of LA) also yields $\delta_{j}^{i}$ ("Identity" in LA):

$$
z_{i j} z^{k i}=\delta_{j}^{k}
$$

The tensors $z_{i j}$ and $z^{i j}$ are used for raising and lowering indices. Raising the second index of $z_{i j}$ by $z^{j k}$ leads to $z_{i}^{\cdot k}$, but it also leads to $\delta_{i}^{\cdot k}$ ! Therefore, $\delta$ and $z$ can be used interchangeably (but then $\delta_{i j}$ no longer has the Kronecker interpretation).

The inner product of two vectors $\mathbf{a}=a^{i} \mathbf{z}_{i}$ and $\mathbf{b}=b^{i} \mathbf{z}_{i}$ is given by

$$
\mathbf{a} \cdot \mathbf{b}=a^{i} \mathbf{z}_{i} \cdot b^{i} \mathbf{z}_{i}=z_{i j} a^{i} b^{j}=a^{i} b_{i}=a_{j} b^{j} .
$$

The contravariant component $a^{i}$ can be extracted by dotting a with $\mathbf{z}^{i}$ :

$$
a^{i}=\mathbf{a} \cdot \mathbf{z}^{i} .
$$

The covariant derivative $\nabla_{i}$, as applied to a typical tensor $T_{\cdot k}^{j}$, is defined as

$$
\nabla_{i} T_{\cdot k}^{j}=\frac{\partial T_{\cdot k}^{j}(z)}{\partial z^{i}}+\Gamma_{i m}^{j} T_{\cdot k}^{m}-\Gamma_{i k}^{m} T_{\cdot m}^{j}
$$

where the Christoffel symbol $\Gamma_{j k}^{i}$ (not a tensor) is defined as

$$
\Gamma_{j k}^{i}=\frac{\partial \mathbf{z}_{j}}{\partial z^{k}} \cdot \mathbf{z}^{i}
$$


When applied to tensors, the covariant derivative yields tensors of one covariant order higher. The covariant derivative reduces to the partial when applied to tensors of order zero, or when applied to general tensors in affine coordinate systems. Covariant differentiation in Euclidean spaces is commutative

$$
\nabla_{i} \nabla_{j}=\nabla_{j} \nabla_{i}
$$

and kills all metrics

$$
\nabla_{m} z_{i j}, \nabla_{m} \delta_{j}^{i}, \nabla_{m} z^{i j}, \nabla_{m} z, \nabla_{m} \mathbf{z}_{i}, \nabla_{m} \mathbf{z}^{i}, \nabla_{m} z_{i j k}, \nabla_{m} z^{i j k}=0 .
$$

7.2. Objects defined on embedded surfaces. Consider a surface $S$ given parametrically in coordinates $\xi^{\alpha}$ as

$$
z^{i}=z^{i}(\xi)
$$

The shift tensor $z_{\alpha}^{i}$ is defined as

$$
z_{\alpha}^{i}=\frac{\partial z^{i}}{\partial \xi^{\alpha}}
$$

It is a tensor with respect to both a change of coordinates in space and a change of coordinates on the surface.

The covariant basis $\xi_{\alpha}$ is defined as

$$
\xi_{\alpha}=\frac{\partial \mathbf{z}}{\partial \xi^{\alpha}}
$$

An application of the chain rule gives an alternative expression

$$
\xi_{\alpha}=z_{\alpha}^{i} \mathbf{z}_{i}
$$

and suggests the interpretation of $z_{\alpha}^{i}$ as a projection operator. Any surface vector $\mathbf{a}=$ $a^{\alpha} \xi_{\alpha}$ can be decomposed with respect to the spatial basis $\mathbf{z}_{i}$ as $a=a^{i} \mathbf{z}_{i}$, where $a^{i}=z_{\alpha}^{i} a^{\alpha}$.

The covariant metric tensor $\xi_{\alpha \beta}$ is defined as

$$
\xi_{\alpha \beta}=\xi_{\alpha} \cdot \xi_{\beta}
$$

and is also expressed as

$$
\xi_{\alpha \beta}=z_{i j} z_{\alpha}^{i} z_{\beta}^{j},
$$

which (running one sentence ahead) can be alternatively written as

$$
z_{\alpha}^{i} z_{i}^{\beta}=\delta_{\alpha}^{\beta} \text {. }
$$

The contravariant metric tensor $\xi^{\alpha \beta}$ is defined as the matrix inverse of $\xi_{\alpha \beta}$ :

$$
\xi_{\alpha \beta} \xi^{\beta \gamma}=\delta_{\alpha}^{\gamma} ; \quad \xi_{\alpha \beta} \xi^{\gamma \alpha}=\delta_{\beta}^{\gamma} .
$$

When it leads to no confusion, the determinant $\left|\xi_{\alpha \beta}\right|$ (not a tensor) is denoted by $\xi$.

The permutation tensors $\varepsilon_{\alpha \beta}$ and $\varepsilon^{\alpha \beta}$ (reusing letter $\xi$ is impossible) are defined as

$$
\varepsilon_{\alpha \beta}=\sqrt{\xi} e_{\alpha \beta}, \quad \varepsilon^{\alpha \beta}=\frac{1}{\sqrt{\xi}} e^{\alpha \beta},
$$

where $e^{12}=-e^{21}=1$ and $e^{11}=e^{22}=0$. 
The normal $N^{i}$ is defined as

$$
N^{i}=\frac{1}{2} z^{i j k} \varepsilon_{\alpha \beta} z_{j}^{\alpha} z_{k}^{\beta} .
$$

It is of unit length, $N^{i} N_{i}=1$, and is orthogonal to the surface $N^{i} z_{i}^{\alpha}=0$.

The tensor $z_{\alpha}^{i} z_{j}^{\alpha}$ is interpreted as the projection onto the surface. This interpretation is firmly justified by the following identity:

$$
z_{\alpha}^{i} z_{j}^{\alpha}=\delta_{j}^{i}-N^{i} N_{j}
$$

The covariant derivative $\nabla_{\gamma}$ as applied to a typical tensor $T_{\cdot k \cdot \beta}^{j \cdot \alpha \cdot}$ is defined as

$$
\nabla_{\gamma} T_{\cdot j \cdot \beta}^{i \cdot \alpha \cdot}=\frac{T_{\cdot j \cdot \beta}^{i \cdot \alpha \cdot}}{\partial \xi \gamma}+z_{\gamma}^{k} \Gamma_{k m}^{i} T_{\cdot j \cdot \beta}^{m \cdot \alpha \cdot}-z_{\gamma}^{k} \Gamma_{k j}^{m} T_{\cdot m \cdot \beta}^{i \cdot \alpha \cdot}+\Gamma_{\gamma \delta}^{\alpha} T_{\cdot j \cdot \beta}^{i \cdot \delta \cdot}-\Gamma_{\gamma \beta}^{\delta} T_{\cdot j \cdot \delta}^{i \cdot \alpha \cdot},
$$

where the Christoffel symbol $\Gamma_{\beta \gamma}^{\alpha}$ (not a tensor) must now be defined traditionally:

$$
\Gamma_{\beta \gamma}^{\alpha}=\frac{1}{2} \xi^{\alpha \omega}\left(\frac{\partial \xi_{\omega \gamma}}{\partial \xi^{\beta}}+\frac{\partial \xi_{\omega \beta}}{\partial \xi^{\gamma}}-\frac{\partial \xi_{\beta \gamma}}{\partial \xi^{\omega}}\right) .
$$

When applied to a surface restriction of a spatial tensor $T_{\cdot j}^{i}$ we have the chain rule

$$
\nabla_{\alpha} T_{\cdot j}^{i}=z_{\alpha}^{k} \nabla_{k} T_{\cdot j}^{i} .
$$

The covariant surface derivative kills many of the metrics (but not the bases):

$$
\nabla_{\lambda} \xi_{\alpha \beta}, \nabla_{\lambda} \delta_{\alpha}^{\beta}, \nabla_{\lambda} \xi^{\alpha \beta}, \nabla_{\lambda} \varepsilon_{\alpha \beta}, \nabla_{\lambda} \varepsilon^{\alpha \beta}=0 .
$$

The chain rule tells us that the covariant surface derivatives kills all spatial metrics:

$$
\nabla_{\gamma} z_{i j}, \nabla_{\gamma} \delta_{j}^{i}, \nabla_{\gamma} z^{i j}, \nabla_{\gamma} z, \nabla_{\gamma} \mathbf{z}_{i}, \nabla_{\gamma} \mathbf{z}^{i}, \nabla_{\gamma} z_{i j k}, \nabla_{\gamma} z^{i j k}=0 .
$$

The curvature tensor $B_{\beta}^{\alpha}$ is defined as

$$
B_{\alpha \beta}=N_{i} \nabla_{\alpha} z_{\beta}^{i} .
$$

Multiplying both sides by $N^{j}$ and applying the projection formula, we get $N^{j} B_{\alpha \beta}=$ $N^{j} N_{i} \nabla_{\alpha} z_{\beta}^{i}=\left(\delta_{i}^{j}-z_{i}^{\gamma} z_{\gamma}^{j}\right) \nabla_{\alpha} z_{\beta}^{i}=\nabla_{\alpha} z_{\beta}^{j}-z_{\gamma}^{j} \nabla_{\alpha} \delta_{\beta}^{\gamma}$. Since the second term is zero, we obtain the formula for differentiating the shift tensor:

$$
\nabla_{\alpha} z_{\beta}^{i}=N^{i} B_{\alpha \beta} .
$$

Once again taking the definition of $B_{\alpha \beta}$ and multiplying both sides by $z_{\beta}^{j}$, we get $z^{j \beta} B_{\alpha \beta}=N_{i} z^{j \beta} \nabla_{\alpha} z_{\beta}^{i}=-z^{j \beta} z_{\beta}^{i} \nabla_{\alpha} N_{i}=\left(-\delta^{i j}+N^{i} N_{j}\right) \nabla_{\alpha} N_{i}=-\nabla_{\alpha} N^{j}+N_{j} N^{i} \nabla_{\alpha} N_{i}$. Since $N^{i} N_{i} \equiv 1$, the second term vanishes and we obtain the formula for differentiating the normal

$$
\nabla_{\alpha} N^{i}=-z^{i \beta} B_{\alpha \beta}
$$

Gauss's Theorema Egregium

$$
\left|B_{. .}\right| \varepsilon_{\nu \mu} \varepsilon_{\alpha \beta}=B_{\alpha \nu} B_{\beta \mu}-B_{\beta \nu} B_{\alpha \mu}
$$

can be interpreted as saying that the tensor $B_{\alpha \beta}$ has only two degrees of freedom (the mean curvature and the intrinsic curvature). 
7.3. The $\delta / \delta \tau$-derivative for moving surfaces. Let the motion of the surface $S_{\tau}$ be described by

$$
S_{\tau}: z^{i}=z^{i}(\tau, \xi)
$$

Define the marker velocity $v^{i}$ (not a tensor) and its projection $v^{\alpha}$ onto the surface (also not a tensor) as

$$
\begin{aligned}
v^{i}(\tau, \xi) & =\frac{\partial z^{i}(\tau, \xi)}{\partial \tau}, \\
v^{\alpha} & =z_{i}^{\alpha} v^{i} .
\end{aligned}
$$

For a tensor of order zero $T$, the derivative $\frac{\delta T}{\delta \tau}$ is defined as [1]

$$
\frac{\delta T(\tau, \xi)}{\delta \tau}=\frac{\partial T(\tau, \xi)}{\partial \tau}-v^{\alpha} \nabla_{\alpha} T
$$

The physical interpretation of this definition is shown in Figure 5 .

The velocity of the interface $C$ is defined as

$$
C=\frac{\delta \mathbf{z}}{\delta \tau} \cdot \mathbf{N}
$$

It is clear from Figure 5 that $\frac{\delta \mathbf{z}}{\delta \tau}$ is normal to the surface, so $\frac{\delta \mathbf{z}}{\delta \tau}=C \mathbf{N}$. We also have

$$
C=v^{i} N_{i}
$$

The definition (108) applies only to scalars and not more general tensors such as $z_{\alpha}^{i}$ and $B_{\beta}^{\alpha}$. For an arbitrary tensor $T_{\cdot j \cdot \beta}^{i \cdot \alpha \cdot}$, the definition of $\delta / \delta \tau$-derivative was given in [2]:

$$
\begin{aligned}
\frac{\delta T_{\cdot j \cdot \beta}^{i \cdot \alpha \cdot}(\tau \cdot \xi)}{\delta \tau}= & \frac{\partial T_{\cdot j \cdot \beta}^{i \cdot \alpha \cdot}(\tau \cdot \xi)}{\partial \tau}-v^{\gamma} \nabla_{\gamma} T_{\cdot j \cdot \beta}^{i \cdot \alpha \cdot} \\
& +v^{m} \Gamma_{m k}^{i} T_{\cdot j \cdot \beta}^{k \cdot \alpha \cdot}-v^{m} \Gamma_{m j}^{k} T_{\cdot k \cdot \beta}^{i \cdot \alpha \cdot}+\nabla_{\gamma} v^{\alpha} T_{\cdot j \cdot \beta}^{i \cdot \gamma \cdot}-\nabla_{\beta} v^{\gamma} T_{\cdot j \cdot \gamma}^{i \cdot \alpha \cdot},
\end{aligned}
$$

where $\nabla_{\gamma} v^{\alpha}$ and $\nabla_{\beta} v^{\gamma}$ are formal applications of the covariant surface derivative to the non-tensor $v^{\alpha}$.

For restrictions of spatial tensors $T_{\cdot j}^{i \cdot}$, we find very useful the chain rule

$$
\frac{\delta T_{\cdot j}^{i \cdot}}{\delta \tau}=\frac{\partial T_{\cdot j}^{i \cdot}}{\partial \tau}+C N^{k} \nabla_{k} T_{\cdot j}^{i \cdot}
$$

The chain rule indicates that the $\frac{\delta}{\delta \tau}$-derivative kills all spatial metrics

$$
\frac{\delta z_{i j}}{\delta \tau}, \frac{\delta \delta_{j}^{i}}{\delta \tau}, \frac{\delta z^{i j}}{\delta \tau}, \frac{\delta z}{\delta \tau}, \frac{\delta \mathbf{z}_{i}}{\delta \tau}, \frac{\delta \mathbf{z}^{i}}{\delta \tau}, \frac{\delta z_{i j k}}{\delta \tau}, \frac{\delta z^{i j k}}{\delta \tau}=0 .
$$

It is also clear for such tensors that $\frac{\delta}{\delta \tau}$ commutes with the covariant space derivative $\nabla_{i}$.

7.4. Properties of $\delta / \delta \tau$. The key properties of the $\delta / \delta \tau$-derivative, sufficient for the derivation of all relationships in the text, are given in the following table:

$$
\begin{aligned}
\frac{\delta z_{\alpha}^{i}}{\delta \tau} & =\nabla_{\alpha}\left(C N^{i}\right), \\
\frac{\delta}{\delta \tau} \delta_{\beta}^{\alpha} & =0, \\
\frac{\delta \xi_{\alpha \beta}}{\delta \tau} & =-2 C B_{\alpha \beta},
\end{aligned}
$$




$$
\begin{aligned}
\frac{\delta \xi^{\alpha \beta}}{\delta \tau} & =2 C B^{\alpha \beta}, \\
\frac{\delta N^{i}}{\delta \tau} & =-z_{\alpha}^{i} \nabla^{\alpha} C, \\
\frac{\delta B_{\beta}^{\alpha}}{\delta \tau} & =\nabla_{\beta} \nabla^{\alpha} C+C B_{\gamma}^{\alpha} B_{\beta}^{\gamma} .
\end{aligned}
$$

Acknowledgements. We thank Michael Person for helping us get up to speed with Mathematica.

\section{REFERENCES}

[1] F.H. Busse, 1974. On the free oscillation of the Earth's inner core. J. Geophys. Res. 79, 753-757.

[2] M.A. Grinfeld, Thermodynamic Methods in the Theory of Heterogeneous Systems. Longman, Sussex, 1991. MR243876 (96e:73010)

[3] J.S. Hadamard. Leçons sur la propagation des ondes et les équations de l'hydrodynamique. A. Hermann, Paris (1903).

[4] H. Jeffreys. The Earth. Cambridge University Press, 5th ed. (1970).

[5] L. D. Landau and E. M. Lifschitz, Quantum Mechanics (Non-Relativistic Theory), 3rd ed. Pergamon Press, Oxford, England (1977). MR0093319 (19:1230k)

[6] Tullio Levi-Civita, The Absolute Differential Calculus (Calculus of Tensors). Dover Publications (1977).

[7] A. J. McConnell. Applications of Tensor Analysis. Dover Publications (1960).

[8] A.B. Migdal. Qualitative Methods in Quantum Theory, W. A. Benjamin, Reading, Mass. (1977). MR0522455(58:25508b)

[9] L. Slichter, 1961. The fundamental free mode of the Earth's inner core. Proc. Natl. Acad. Sci. U.S.A. 47, 186-190.

[10] A.M.K. Szeto and S. Xu, 1997. Gravitational Coupling in a Triaxial Ellipsoidal Earth. J. Geophys. Res., vol. 102, 27651-27657.

[11] T.Y. Thomas. Plastic flow and fracture in solids. Academic Press, New York (1961). MR.127630 (23:B675)

[12] Hermann Weyl. Space time matter. Dover Publications (1984).

[13] S. Wolfram. The Mathematica Book, 5th ed. Wolfram Media (2003). MR.1404696 (97d:68001) 